\title{
On the graded Betti numbers for large finite subsets of curves
}

\author{
by E. BALlico (Trento)
}

\begin{abstract}
We prove a recent conjecture of S. Lvovski concerning the periodicity behaviour of top Betti numbers of general finite subsets with large cardinality of an irreducible curve $C \subset \mathbb{P}^{n}$.
\end{abstract}

Introduction. In [5], S. Lvovski made a nice Periodicity Conjecture (see [5], Conjecture 1.2, or the case $B=\emptyset$ of our theorem below for its statement) about the graded Betti numbers of general subsets with large cardinality of a fixed irreducible curve $C \subset \mathbb{P}^{n}$. He proved it in some cases (e.g. for the lower Betti diagram ([5], Prop. 5.1) or if $C$ is a rational normal curve). He raised also a generalization of this conjecture (see [5], Conjecture 1.3) which inspired the statement of our theorem. We will not use the methods introduced in [5] and hence we will be able to work over an uncountable algebraically closed field $\mathbf{K}$ with arbitrary characteristic.

To state our results we need to introduce the following notations. Set $\mathbb{P}^{n}:=\operatorname{Proj}(R)$ with $R:=\mathbf{K}\left[T_{0}, \ldots, T_{n}\right]$. For any closed subscheme $Z$ of $\mathbb{P}^{n}$, let $\mathbf{I}_{Z} \subset R$ be its homogeneous ideal and $L_{*}(Z)$ its minimal graded free resolution. Hence $L_{0}(Z)=R$ and $L_{m}(Z)=0$ for $m>n$. If $L_{i}=$ $\bigoplus_{j} R(-i-j)^{b_{i j}(Z)}$, then the non-negative integers $b_{i j}(Z)$ will be called the graded Betti numbers of $Z$. Our notations for graded Betti numbers agree with those adopted by the program Macaulay and in [5]. Set $\delta(Z):=\max \{j$ : there is $i$ with $\left.b_{i j}(Z) \neq 0\right\}$. Call $\delta(Z)$ the index of regularity of $Z$.

For a very good introduction to the Koszul cohomology of finite sets and linearly normal curves, see [2], §1, or [3], Introduction and $\S 1$, or [4], §1. We only need the following fact. Let $\Omega^{j}, 0 \leq j \leq n$, be the sheaf of exterior $j$-forms on $\mathbb{P}^{n}$. Fix integers $n, i, k$ with $n \geq 2,1 \leq i \leq n$ and $k>0$. Let $A \subseteq \mathbb{P}^{n}$ be a closed subscheme such that $h^{i}\left(A, \mathcal{O}_{A}(z)\right)=0$ for every $i>0$

1991 Mathematics Subject Classification: 14N05, 14H99, 13D40, 13P99.

Key words and phrases: Koszul cohomology, irreducible curve, Betti diagram. 
and every $z \geq k-i$; this condition is satisfied if $\operatorname{dim}(A)=0$. We have $b_{i j}(A)=0$ for every $j \geq k$ if and only if $h^{1}\left(\mathbb{P}^{n}, \Omega^{i}(i+k) \otimes \mathbf{I}_{A}\right)=0$.

Theorem. Let $C \subset \mathbb{P}^{n}$ be an irreducible curve and $B \subset C$ an effective (or empty) Cartier divisor of C. Set $d:=\operatorname{deg}(C)$. Let $\left\{P_{i}\right\}_{i \in \mathbb{N}}$ be a generic sequence of points of $C$ and for every $m>0$ set $X_{m}:=B \cup\left\{P_{1}, \ldots, P_{m}\right\}$. Set $t_{m}:=\min \left\{j>\delta(C)\right.$ : there is $i$ with $\left.b_{i j}\left(X_{m}\right) \neq 0\right\}$. Then there in an integer $m^{\prime}$ such that for all integers $m \geq m^{\prime}$ we have $t_{m+d}=t_{m}+1$ and if $j \geq t_{m}$, then $b_{i, j+1}\left(X_{m+d}\right)=b_{i j}\left(X_{m}\right)$ for all $i$. Moreover, the periodic pattern appearing for large $m$ in the Betti diagram of $X_{m}$ depends only on the integers $d, g:=p_{a}(C), \delta(C)$ and $b:=\operatorname{length}(B)$.

Indeed our proof of this theorem will give some information on the graded Betti numbers of $X_{m}$ for large $m$. Furthermore, the proof will show that the cases $m+b+1-g \equiv 0 \bmod d$ and $m+b+1-g \equiv 1 \bmod d$ are "easier" than the cases with $m+b+1-g \equiv i \bmod d$ and $2 \leq i<d$.

The author was partially supported by MURST and GNSAGA of CNR (Italy) and by Max-Planck-Institut für Mathematik in Bonn. He wants to thank the Max-Planck-Institut for excellent working atmosphere.

The proof. If $P_{i} \in C_{\mathrm{reg}}, i \geq 1$, and $m \geq 1$, set $Y\{m\}:=\sum_{1<i<m} P_{i}$ and $X\{m\}:=B+Y\{m\}$. Hence $Y_{m}:=\bigcup_{1<i<m} P_{i}$ and $X_{m}:=\bar{B} \cup Y_{m}$ are 0-dimensional closed subschemes of $C, Y\{m\}$ is the effective degree $m$ Cartier divisor of $C$ associated with $Y_{m}$ and $X\{m\}$ is the effective degree $m+b$ Cartier divisor of $C$ associated with $X_{m}$. Note that if $m \geq g$ for general $P_{i}$ the line bundle $\mathcal{O}_{C}(Y\{m\})$ (resp. $\left.\mathcal{O}_{C}(X\{m\})\right)$ is a general line bundle of degree $m$ (resp. degree $b+m$ ) on $C$. A general $L \in \operatorname{Pic}^{w}(C)$ has $h^{1}(C, L)=0$ (resp. $h^{0}(C, L) \neq 0$, resp. it is spanned) if and only if $w \geq g-1$ (resp. $w \geq g$, resp. $w \geq g+1)$. Hence for every integer $z$ we have $h^{1}\left(C, \mathcal{O}_{C}(z)(-X\{m\})\right)=$ 0 if and only if $z d \geq b+m+g-1$ and $h^{0}\left(C, \mathcal{O}_{C}(z)(-X\{m\})\right) \neq 0$ if and only if $z d \geq b+m+g$. Furthermore, $\mathcal{O}_{C}(z)(-X\{m\})$ is spanned by its global sections if and only if $z d \geq b+m+g+1$, i.e. if and only if $h^{0}\left(C, \mathcal{O}_{C}(z)(-X\{m\})\right)=z d-b-m+1-g \geq 2$.

Fix an integer $i$ with $0 \leq i<d$. We are interested in the integers $m$ with $m \equiv i \bmod d$. Let $\alpha(i, m)$ be the first integer $>\delta(C)$ with $m \geq d$ and $d \alpha(i, m)-m-b \geq g$. The proof of our theorem will show that the inequality $m \geq d$ can be easily weakened. For general $X_{m}$ we have $h^{0}\left(\mathbb{P}^{n}, \mathbf{I}_{X_{m}}(t)\right)=$ $h^{0}\left(\mathbb{P}^{n}, \mathbf{I}_{C}(t)\right)$ if and only if $t<\alpha(i, m)$. Hence $t_{m}=\alpha(i, m)$. Note that $\alpha(i, m+d)=\alpha(i, m)+1$. Thus we have proved that for general $\left\{P_{i}\right\}_{i \in \mathbb{N}}$ we have $t_{m+d}=t_{m}+1$, i.e. the first assertion of the theorem.

Furthermore, the restriction map $H^{0}\left(\mathbb{P}^{n}, \mathcal{O}_{\mathbb{P}^{n}}(t)\right) \rightarrow H^{0}\left(X_{m}, \mathbf{I}_{X_{m}}(t)\right)$ is surjective if and only if $t \geq \alpha(i, m)$. By Castelnuovo-Mumford's regularity theorem, we have $\alpha(i, m) \leq \delta\left(X_{m}\right) \leq \alpha(i, m)+1$. 
If $0 \leq d \alpha(i, m)-b-m-g+1 \leq 1$, then $\mathbf{I}_{X_{m}}$ cannot be generated by forms of degree $\leq \alpha(i, m)$ and hence we have $\delta\left(X_{m}\right)=\alpha(i, m)+1$. The last part of our proof concerning the values of $h^{0}\left(\mathbb{P}^{n}, \Omega^{t}(t+\alpha(i, m)) \otimes \mathbf{I}_{X_{m}}\right)$ and $h^{0}\left(\mathbb{P}^{n}, \Omega^{t}(t+\alpha(i, m)+1) \otimes \mathbf{I}_{X_{m+d}}\right)$ will show even in this case the equality $b_{i, j+1}\left(X_{m+d}\right)=b_{i j}\left(X_{m}\right)$, completing the proof of our theorem in this case.

If $2 \leq d \alpha(i, m)-b-m-g+1<d$, then $\alpha(i, m)$ is the first integer $t$ such that the part of degree $t$ of $\mathbf{I}_{X_{m}}$ has $X_{m}$ as scheme-theoretic 0-locus. If for all integers $m \geq m^{\prime}$ with $m \equiv i \bmod d$ we have $\delta\left(X_{m}\right)=$ $\alpha(i, m)+1$, then we have proved the assertion on the index of regularity in the statement of the theorem for the integers $m$ in the congruence class of $i$ modulo $d$. Hence we may assume the existence of an integer $m \geq m^{\prime}$ with $m \equiv i \bmod d$ such that $\delta\left(X_{m}\right)=\alpha(i, m)$.

It is sufficient to prove that $\delta\left(X_{m+d}\right)=\alpha(i, m)$. As explained before the statement of the theorem, the assertion $\delta\left(X_{m}\right)=\alpha(i, m)\left(\operatorname{resp} . \delta\left(X_{m+d}\right)=\right.$ $\alpha(i, m)+1)$ is equivalent to the fact that for all integers $t$ with $1 \leq t \leq n$ we have $h^{1}\left(\mathbb{P}^{n}, \Omega^{t}(t+\alpha(i, m)) \otimes \mathbf{I}_{X_{m}}\right)=0$ (resp. $h^{1}\left(\mathbb{P}^{n}, \Omega^{t}(t+\alpha(i, m)+\right.$ $\left.1) \otimes \mathbf{I}_{X_{m+d}}\right)=0$ ). By semicontinuity it is sufficient to show the vanishing of these cohomology groups for a very special finite subset, $X_{m+d}$, of $C$ with $B \subseteq X_{m} \subseteq X_{m+d}$ and $\operatorname{card}\left(X_{m+d}\right)=\operatorname{card}\left(X_{m}\right)+d=b+m+d$. We take a general hyperplane $H$ and prove that $h^{1}\left(\mathbb{P}^{n}, \Omega^{t}(t+\alpha(i, m)+1) \otimes \mathbf{I}_{X_{m+d}}\right)=0$ when $X_{m+d}$ is the union of $X_{m}$ and of the hyperplane section $H \cap C$ of $C$. Note that $\Omega^{t}(t+\alpha(i, m)+1) \mid H \cong \Omega_{H}^{t}(t+\alpha(i, m)+1) \oplus \Omega_{H}^{t-1}(t+\alpha(i, m))$.

Since we assumed $\alpha(i, m) \geq d$, the graded Betti numbers of $C \cap H$ in $H$ are all lower than $c(i, m)$. Thus again by Koszul cohomology we have $H^{1}\left(H, \mathbf{I}_{C \cap H, H} \otimes \Omega^{t}(t+\alpha(i, m)+1) \mid H\right)=0$. Thus we obtain $\delta\left(X_{m+d}\right)=$ $\alpha(i, m)+1$ using the following short exact sequence:

$$
\begin{aligned}
0 \rightarrow \mathbf{I}_{X_{m}} \otimes \Omega^{t}(t+\alpha(i, m)) & \rightarrow \mathbf{I}_{X_{m+d}} \otimes \Omega^{t}(t+\alpha(i, m)+1) \\
& \rightarrow \mathbf{I}_{C \cap H, H} \otimes\left(\Omega^{t}(t+\alpha(i, m)+1) \mid H\right) \rightarrow 0 .
\end{aligned}
$$

The top graded Betti numbers of $X_{m}$ (resp. $X_{m+d}$ ) are uniquely determined by the ordered set of $t+1$ integers $h^{0}\left(\mathbb{P}^{n}, \Omega^{t}(t+\alpha(i, m)) \otimes \mathbf{I}_{X_{m}}\right)$ (resp. $\left.h^{0}\left(\mathbb{P}^{n}, \Omega^{t}(t+\alpha(i, m)+1) \otimes \mathbf{I}_{X_{m+d}}\right)\right), 0 \leq t \leq n$, and hence we obtain in both cases the statements on $\delta\left(X_{m}\right)-\delta\left(X_{m+d}\right)$ and on the graded Betti numbers of $X_{m}$ and $X_{m+d}$.

\section{References}

[1] M. Green, Koszul cohomology, J. Differential Geom. 19 (1984), 125-171.

[2] - Koszul cohomology and geometry, in: Lectures on Riemann Surfaces (Trieste, 1987), World Sci., 1989, 177-200.

[3] M. Green and R. Lazarsfeld, Some results on the syzygies of finite sets and algebraic curves, Compositio Math. 67 (1988), 301-314. 
[4] R. Lazarsfeld, A sampling of vector bundles techniques in the study of linear series, in: Lectures on Riemann Surfaces (Trieste, 1987), World Sci., 1989, 500-559.

[5] S. Lvovski, On graded Betti numbers for finite subsets of curves, preprint 97-31, Max-Planck-Institut.

Department of Mathematics

University of Trento

38050 Povo (TN), Italy

E-mail: ballico@science.unitn.it

Reçu par la Rédaction le 17.11.1997

Révisé le 16.2.1998 